\title{
Feature extraction of On-Load Tap-Changer Based on Improved EMD Energy Spectrum
}

\author{
Huang Wenquan*
}

School of Information Science and Engineering, Huaqiao University, Xiamen, 361021, China

\begin{abstract}
On-Load Tap-Changer is an important device with high failure rate in power systems. This paper proposes an improved EMD energy spectrum feature extraction method based on vibration signals. It divides the entire OLTC shifting process into two sections, and extracts their characteristic energy spectrum. Analysis of sample data shows that this method can more effectively distinguish the state of OLTC.
\end{abstract}

\section{Introduction}

The On-Load Tap-Changer (OLTC) plays an important role in the process of the transformer adjusting the reactive power flow and stabilizing the center voltage of the load. The reliability of the OLTC is directly related to the stability of the transformer and the power system. According to statistics from foreign countries, OLTC faults account for $41 \%$ of faults in load-regulating transformers ${ }^{[5][7]}$. Therefore, the online fault diagnosis of OLTC is a problem that needs to be solved urgently.

The vibration signal is generated during the shifting process of OLTC. When OLTC has hidden troubles or faults, its vibration signal will change. By extracting certain feature from the vibration signal, it can diagnose the status of OLTC, timely repair and improve the reliability of the equipment. Scholars Rivas et al. used hilbert transform and discrete wavelet transform to analyze the vibration signals of different parts of OLTC, and obtained the peak time and amplitude information of the signal envelope ${ }^{[1]}$. Scholars Zhao Tong and others reconstructed the vibration signal in high-dimensional space, and defined the phase point spatial distribution coefficient to identify the normal and fault states of OLTC

\footnotetext{
* Corresponding author: peter81015@hqu.edu.cn
} 
${ }^{[7][8]}$.Ma Hongzhong and Zhang Huifeng of Hohai University proposed the diagnosis using EMD-HT time-frequency analysis algorithm, EMD entropy and wavelet entropy ${ }^{[2][3]}$. These methods solve the OLTC feature extraction problem to a certain extent, but many methods are limited to feature extraction for a few hundred milliseconds of the in-position section, ignoring the feature information contained in the stationary section.

In this paper, the entire vibration signal of OLTC shifting process is obtained. After the signal pre-processing, it is divided into a stationary section and an in-position section and the energy spectrum of two sections are calculated using the EMD method. The analysis of these energy spectrum samples shows that these method can better identify some failures, such as mechanism jam.

\section{EMD energy spectrum algorithm}

\subsection{EMD energy spectrum algorithm}

The EMD method assumes that any signal can be decomposed into multiple IMF components and residuals as shown in Equation 1.

(1) Perform EMD method for vibration signal data $S(t)$. Each IMF component can be regarded as the component of the original signal in a certain frequency band.

$$
s(t)=\sum_{i=1}^{n} c_{i}(t)+r_{n}(t)
$$

$r_{n}(\mathrm{t})$ is the residual function, which represents the average trend of the signal.

(2) Define the IMF energy as follow, and calculate the energy of each IMF :

$$
E(i)=\frac{1}{N} \sum_{i=1}^{N}\left|c_{i}(t)\right|^{2}
$$

Where, $N$ is the length of the IMF. The total signal energy is:

$$
E=\sum_{i=1}^{M} E(i)
$$

Take $\varepsilon_{k}(i)=E_{k} / E$, then $\sum_{k} \varepsilon_{k}=1$.Define EMD energy entropy as:

$$
H_{k}=-\sum_{i=1}^{N} \varepsilon_{k}(i) \log \left|\varepsilon_{k}(i)\right|
$$

(3) Construct the EMD energy spectrum

Based on the EMD energy entropy by Equation (4), let $H=\sqrt{\sum\left|H_{k}\right|^{2}}$ and normalize the EMD energy spectrum of the stationary section. 


$$
T_{1}^{\prime}=\left[\frac{H_{11}}{H_{1}}, \frac{H_{12}}{H_{1}}, \mathrm{~L}, \frac{H_{1 k}}{H_{1}}\right]
$$

(4) According to the method of steps (1)-(3), the EMD energy spectrum of the stationary section $T_{1}^{\prime}$ and the energy spectrum of the in-position section $T_{2}^{\prime}$ are obtained. Therefore, the EMD energy spectrum of the OLTC shifting process $T$ can be defined as:

$$
T=\left[T_{1}^{\prime}, T_{2}^{\prime}\right]=\left[\frac{H_{11}}{H_{1}}, \frac{H_{12}}{H_{1}}, \mathrm{~L}, \frac{H_{1 k}}{H_{1}}, \frac{H_{21}}{H_{2}}, \frac{H_{22}}{H_{2}}, \mathrm{~L}, \frac{H_{2 \mathrm{k}}}{H_{2}}\right]
$$

\subsection{Calculate correlation of the EMD energy spectrum}

Considering that the energy spectrum has been normalized, the Pearson correlation coefficient is used to determine the correlation between different signal samples. The Pearson correlation coefficient is defined as:

$$
\rho_{x, y}=\frac{E\left(\left(x-u_{x}\right)\left(y-u_{y}\right)\right)}{\sigma_{x} \sigma_{y}}=\frac{E(x y)-E(x) E(y)}{\sqrt{E\left(x^{2}\right)-E^{2}(x)} \sqrt{E\left(y^{2}\right)-E^{2}(y)}}
$$

Where $\mathrm{x}$ and $\mathrm{y}$ are the energy spectrum of the samples, $E(x)$ and $E(y)$ are the mean of them. If the feature extraction method is effective, the correlation in the same state will high and the correlation in different states will low. For this article, when $\rho>0.95$, the samples are considered to be similar, and when $\rho<0.85$, the samples are considered different.

\section{OLTC vibration signal preprocessing}

The accelerometer sensor is installed on the head end cap of OLTC ZVIII350. The vibration signal of OLCT collected by the accelerometer is sampled by the data collector and uploaded to the data processing software on the PC in the experiment.
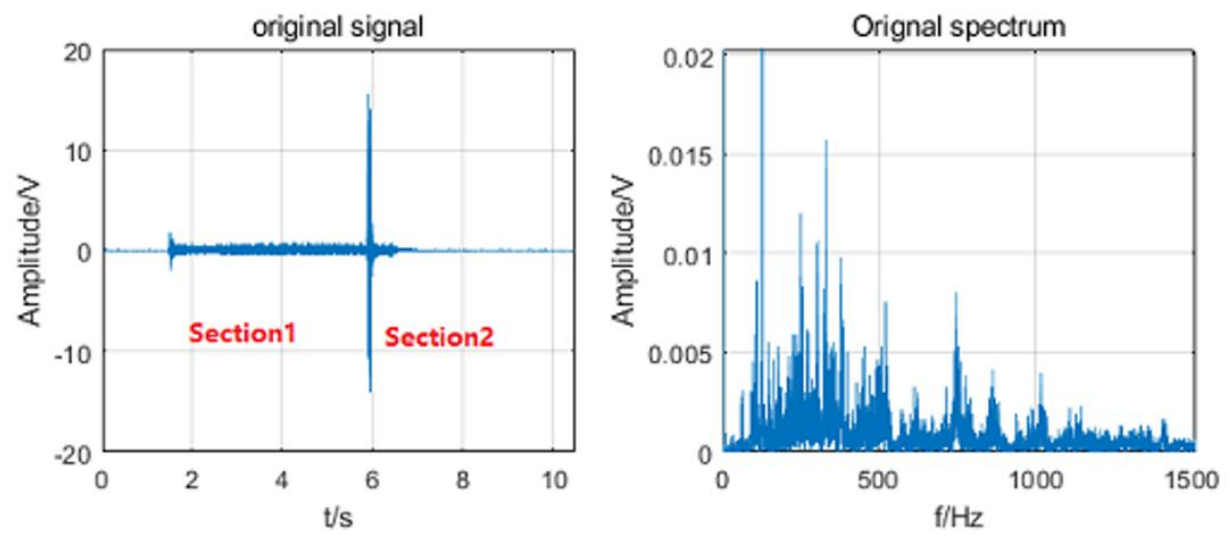

Figure 1. Original vibration waveform and signal spectrum 
From the related literature ${ }^{[6]}$ and the signal spectrum in the right part of Figure 1, it can be found that the energy is mainly concentrated at $50 \mathrm{~Hz}-1 \mathrm{kHz}$. Combining the principle of OLTC and the characteristics of the vibration signal, the entire shifting process can be divided into two section: the stationary section and the in-position section. The stable section is mainly the external electric mechanism driving the spring to store energy. At this stage, the vibration signal is relatively stable and the amplitude is small. The in-position section is the release of the over-dead point of the energy storage mechanism, the switching component operates according to predetermined requirements, and the contacts are switched on and off to achieve gear switching, and the vibration signal changes suddenly.

Taking the signal samples in the normal state as an example, at this time, the OLTC is increased from 1 st gear to 2 nd gear. The vibration signals of the stationary section and the in-place section are solved by empirical mode decomposition to obtain IMF component, and the odd components are shown in the figure below.
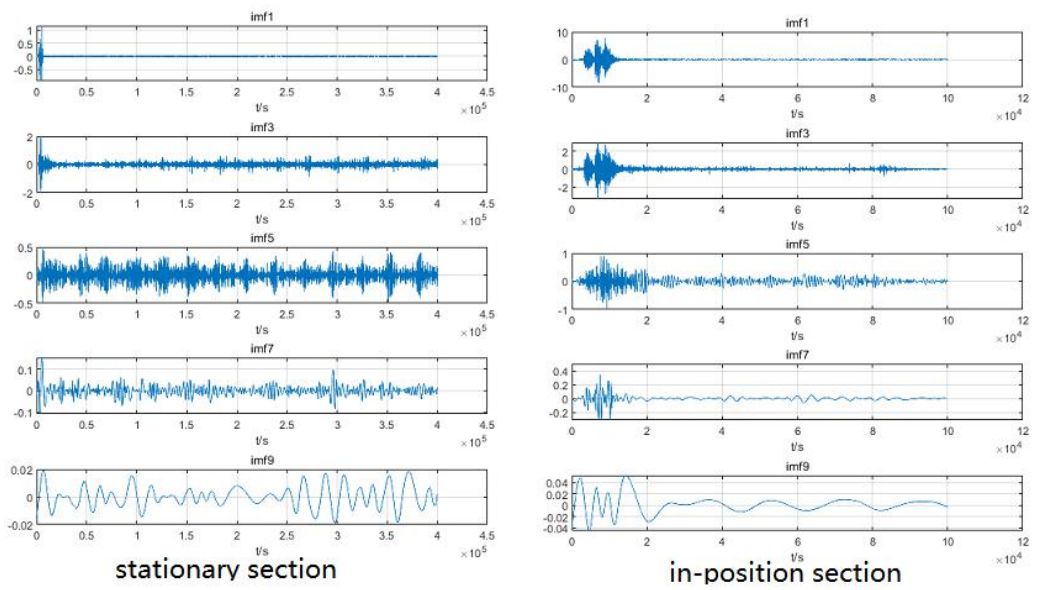

Figure 2. EMD diagram for two section

As shown by the IMF9 waveform in Figure 2, the components IMF8-IMF10 have obvious modal aliasing, and their signal amplitude are only one tenth of other components. Therefore, the characteristic energy spectrum of OLTC is composed of the first 7 IMF components of the stationary section and the in-place section, that is, a total of 14 energy Energy entropy form the EMD characteristic energy spectrum.

\section{Energy spectrum extraction and analysis}

The OLTC vibration experiment obtained a total of 192 sets of vibration signal samples, of which 97 groups were in normal state, 50 groups were mechanism jam, and 45 groups were spring breakage. Five signal samples are taken for each state, and the Pearson correlation coefficient is used for data analysis. 


\subsection{EMD energy spectrum in normal state}

The energy spectrum of five samples in the normal state is shown in the figure below.

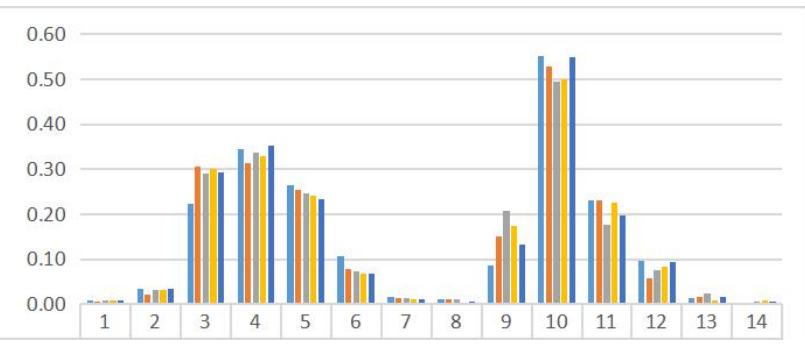

Figure 3. EMD energy spectrum in normal state

It can be seen that the distribution characteristics of the energy spectrum of vibration signal are consistent. The energy is mainly concentrated in the IMF $3 \approx 0.29$,IMF $4 \approx 0.32$, IMF $5 \approx 0.24$ in the stationary section, and the IMF9 $\approx 0.15$, IMF $10 \approx 0.52$, IMF $11 \approx 0.2$ and IMF12 $\approx 0.1$, etc in in-position section. The table 1 shows the correlation of the five samples in normal state is greater than 0.95 , and the similarity of the samples is high.

Table 1 The correlation of EMD energy spectrum in normal state

\begin{tabular}{|c|c|c|c|c|c|}
\hline Correlation & Normal 1 & Normal 2 & Normal 3 & Normal 4 & Normal 5 \\
\hline Normal 1 & 1.000 & 0.978 & 0.985 & 0.960 & 0.972 \\
\hline Normal 2 & 0.978 & 1.000 & 0.992 & 0.988 & 0.996 \\
\hline Normal 3 & 0.985 & 0.992 & 1.000 & 0.987 & 0.992 \\
\hline Normal 4 & 0.960 & 0.988 & 0.987 & 1.000 & 0.994 \\
\hline Normal 5 & 0.972 & 0.996 & 0.992 & 0.994 & 1.000 \\
\hline
\end{tabular}

\subsection{EMD energy spectrum of mechanism jam}

The energy spectrum of signal samples under jam conditions is shown below. 


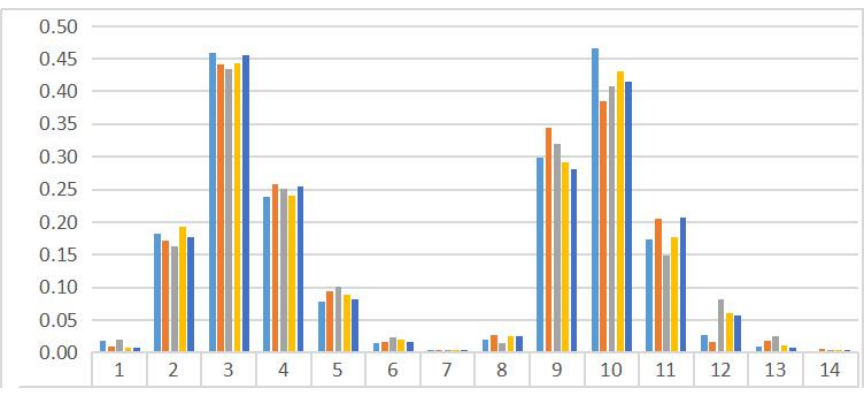

Figure 4. EMD energy spectrum of mechanism jam

The figure shows that the characteristics of the energy spectrum of the mechanism jam faults have good consistency. The corresponding frequency of the IMF component is from high to low. Compared with the normal state, the energy of the high-frequency vibration signal is increased. The IMF2 ratio of the stationary section has increased significantly to 0.17 , the IMF3 ratio has increased to 0.45 , and IMF4 has decreased slightly to 0.25 . The IMF9 ratio of the in-position section increased to 0.3 and IMF10 decreased to 0.4.The correlation of the characteristic energy spectrum of samples is also above 0.95 .

Compared with the same state, the correlation between different states are less than 0.85 in Table 2 below, which indicates that it can distinguish between normal state and mechanism jam.

Table 2 The energy spectrum correlation between mechanism jam and normal state

\begin{tabular}{|c|c|c|c|c|c|}
\hline Correlation & Jam1 & Jam 2 & Jam 3 & Jam 4 & Jam 5 \\
\hline Normal 1 & 0.727 & 0.684 & 0.715 & 0.725 & 0.726 \\
\hline Normal 2 & 0.828 & 0.798 & 0.818 & 0.824 & 0.827 \\
\hline Normal 3 & 0.818 & 0.779 & 0.882 & 0.816 & 0.816 \\
\hline Normal 4 & 0.818 & 0.826 & 0.852 & 0.844 & 0.842 \\
\hline Normal 5 & 0.843 & 0.821 & 0.843 & 0.843 & 0.847 \\
\hline
\end{tabular}

\subsection{The effectiveness of the stationary energy spectrum}

In order to verify the effectiveness of the method, we take the normal state and the mechanism jam as examples, as shown in the Table 3.

Table 3.Comparison of correlation before and after adding stationary features 


\begin{tabular}{|c|c|c|c|c|c|c|c|c|c|c|}
\hline & \multicolumn{3}{|c|}{ without the stationary section } & \multicolumn{5}{c|}{ with the stationary section } \\
\hline Correlatio & & & & & & & & & & \\
$\mathrm{n}$ & Jam 1 & Jam 2 & Jam 3 & Jam 4 & Jam 5 & Jam 1 & Jam 2 & Jam 3 & Jam 4 & Jam 5 \\
\hline Normal 1 & 0.862 & 0.757 & 0.805 & 0.860 & 0.867 & 0.727 & 0.684 & 0.715 & 0.725 & 0.726 \\
\hline Normal 2 & 0.928 & 0.849 & 0.876 & 0.924 & 0.930 & 0.828 & 0.798 & 0.818 & 0.824 & 0.827 \\
\hline Normal 3 & 0.907 & 0.809 & 0.942 & 0.905 & 0.905 & 0.818 & 0.779 & 0.882 & 0.816 & 0.816 \\
\hline Normal 4 & 0.907 & 0.900 & 0.941 & 0.968 & 0.964 & 0.818 & 0.826 & 0.852 & 0.844 & 0.842 \\
\hline Normal 5 & 0.942 & 0.872 & 0.904 & 0.944 & 0.949 & 0.843 & 0.821 & 0.843 & 0.843 & 0.847 \\
\hline
\end{tabular}

It can be seen that the correlation between different states is obviously reduced after the characteristic energy spectrum of the stationary section is supplemented,which indicates that the characteristics energy spectrum of the stationary section is correct and effective.

It should be noted that some states such as contact failure only occur in the in-position section, and the characteristics of the stationary section are not affected. In this case, there will also be a situation where the correlation is slightly increased after adding the characteristics of the stationary section.

\section{Conclusion}

Aiming at the problem that the original OLTC fault diagnosis only focused on the vibration signal of the in-position section, this paper proposes to increase the characteristic energy spectrum of the stationary section as a supplement, and together with the in-position section's characteristic, form the EMD energy spectrum. Without increasing the number of sensors and the complexity of the algorithm, the effectiveness of state feature extraction is improved. The research in this paper shows that the EMD energy spectrum in the same state has regularity and high similarity, but the EMD energy spectrum in different states has obvious differences.

\section{Funding}

The authors disclosed receipt of the following financial support for the research, authorship, and/or publication of this article: Xiamen Science and Technology Plan Project 
(Project No.3502Z20193032), and Natural Science Foundation of Fujian Province, China (Project No. 2019J01054).

\section{References}

1. Rivas-Trujillo E, Edwin Laniado-Jácome, José Luis San-Román, et al. Characterizing the diverter switch of a load tap changer in a transformer using wavelet and modal analysis[J]. Engineering Structures, 2010, 32(10):3011-3017.

2. Zhang Huifeng, Ma Hongzhong, Chen Kai, et al. Fault diagnosis of transformer on-load tap-changer based on time-frequency analysis of vibration signal emd-ht [j]. High Voltage Apparatus, 2012, 48 (1): 76-81.

3. Gao Peng, Ma Hongzhong, Zhang Huifeng, et al. Comparison of emd entropy and wavelet entropy of tap-changer vibration signal [j]. Journal of Electric Power Systems and Automation, 2012, 24 (4): 48-53.

4. Wang Chunliang, Li Kai, Ma Hongzhong, et al. Feature recognition of oltc vibration signals based on wp_svd noise reduction [j]. Journal of Electric Power Systems and Automation, 2012, 24 (5): 36-41.

5. Wang Mengyun. Statistical Analysis of $110 \mathrm{kV}$ and Above Transformer Accidents in 2004 [J]. Electric Power Equipment, 2005, 6 (11): 31-34..

6. Hong Xiang, Ma Hongzhong, Gao Peng, et al. Fault diagnosis of loose contact of on-load tap-changer based on EEMD [J]. HUA DIAN TECHNOLOGY, 2012, 34 (1): $12-15$.

7. Zhao Tong. Research on on-line monitoring and fault diagnosis of on-load tap-changer mechanical status [d]. Shandong University, 2008.

8. Zhao Tong, Li Qingmin, Chen Ping. Dynamic analysis method for feature extraction of oltc vibration signals [j]. Journal of Electrical Engineering and Technology, 2007, 22 (1): 41-46. 